\title{
MMP-2 and MMP-9 are prominent matrix metalloproteinases during atherosclerosis development in the Ldlr $^{-/} A$ pob $^{100 / 100}$ mouse
}

\author{
DICK WÅGSÄTER ${ }^{1}$, CHAOYONG ZHU $^{1}$, JOHAN BJÖRKEGREN ${ }^{2}$, JOSEFIN SKOGSBERG $^{2}$ and PER ERIKSSON ${ }^{1}$ \\ ${ }^{1}$ Atherosclerosis Research Unit, Center for Molecular Medicine, Department of Medicine and ${ }^{2}$ The Cardiovascular \\ Genomics Group, Department of Medical Biochemistry and Biophysics, Karolinska Institute, Solna, Stockholm, Sweden
}

Received February 9, 2011; Accepted March 23, 2011

DOI: $10.3892 / \mathrm{ijmm} .2011 .693$

\begin{abstract}
Matrix-degrading proteases capable of degrading components of the extracellular matrix may play an important role in development and progression of atherosclerotic lesions. In the present study, we used the $L d l r^{-/-} A p o b^{100 / 100}$ mouse model, which has a plasma lipoprotein profile similar to that of humans with atherosclerosis, to study the expression of matrix metalloproteinases (MMPs) during early stages of atherosclerosis development. We analyzed the expression of 11 proteases and three protease inhibitors in 5- to 40-week-old $L d l r^{-1-} A p o b^{100 / 100}$ mice. Expression and activity of MMP-2 and MMP-9 was increased in advanced atherosclerotic lesions followed by macrophage infiltration as shown by real-time PCR, gel-based and in situ zymography and immunohistochemistry. Expression of other investigated MMPs did not increase during disease progression. However, the mRNA expression of MMP-8 and MMP-13 was down-regulated, which could explain the relatively high amount of collagen observed in the vessels in this model. In conclusion, low proteolytic expression at early stages of atherogenesis and a limited repertoire of proteolytic enzymes were associated with the progression of atherosclerosis in $\mathrm{Ldlr}^{-/} \mathrm{Apob} \mathrm{b}^{100 / 100}$ mice. The study suggests that MMP-2 and MMP-9 are the main proteases involved in atherogenesis in this mouse model.
\end{abstract}

\section{Introduction}

Atherosclerosis is a lipid-driven chronic inflammatory process within vessel walls of large arteries (1). The process involves remodeling of the extracellular matrix (ECM), resulting in

Correspondence to: Dr Dick Wågsäter, Atherosclerosis Research Unit, Center for Molecular Medicine, L8:03, Karolinska Institute, Solna, S-171 76 Stockholm, Sweden

E-mail: dick.wagsater@ki.se

Key words: protease, apolipoprotein B 100, extracellular matrix, atherosclerosis atherosclerotic plaque formation. Matrix metalloproteinases (MMPs) are a large family of proteases that can degrade all components of the ECM. MMPs are highly expressed in atherosclerotic plaques and perturbed proteolytic activity has been implicated in atherogenesis and the precipitation of acute coronary syndromes.

Studies in mice have suggested that different members of the MMP family may exert divergent effects during the atherosclerotic process (2). MMPs are proposed to enhance migration and proliferation of smooth muscle and inflammatory cells during early stages of the atherosclerotic process. In advanced plaques, inflammation-derived proteolytic activity may weaken the plaque, leading to its instability. Deficiencies of different MMPs in combination with Apolipoprotein (Apo) E deficiency has indicated that plaque formation differs widely depending on which MMP is deleted (3). For example, plaques are significantly larger in ApoE/MMP-3 and ApoE/ MMP-9 double knockouts as compared to controls and both knockouts exhibit cellular compositional changes indicative of an unstable plaque phenotype. The lesion size is reduced in ApoE/MMP-12 double knockouts with an increased smooth muscle cell and reduced macrophage content in the plaque, indicating a stable plaque phenotype. In humans, plasma concentrations of different MMPs appear to vary depending on clinical status. For example, we have shown that plasma concentrations of MMP-2 were significantly decreased in patients with unstable coronary artery disease (UCAD) compared with patients with stable CAD and healthy controls (4). Similarly, plasma concentrations of MMP-3 were 3-fold higher in the control group than in the group of UCAD patients. In contrast, MMP-7 concentrations were increased by $50 \%$ in unstable CAD patients compared to controls (4). Plasma MMP-9 concentration has been identified as a predictor of cardiovascular mortality in patients with CAD (5). Taken together, these and other data implicate both pro- and anti-atherogenic properties of the different members of the MMP family during the atherosclerotic process (2).

Most research on MMP expression during atherosclerosis has been conducted at late stages of the disease using biopsies from human atherosclerotic tissues or mice overexpressing or deficient in different MMPs. In the present study, we analyzed the expression and activity of several matrix-degrading 
proteases before and during early stages of lesion formation in $\mathrm{Ldlr}^{-1} \mathrm{Apob}^{100 / 100}$ mice. In this model, most of the cholesterol is carried in ApoB100-containing LDL, similarly to the human situation, which makes this model suitable for the study of atherosclerosis (6-9).

\section{Materials and methods}

Animal model. The $L d l r^{-/} A p o b^{100 / 100}$ Mx1Cre Mttp flox/flox mouse on a C57bl/6 background was used without activating the Cre-lox system (Mx1Cre Mttp $\left.{ }^{\text {flox/flox }}\right)$, and therefore, is referred to as the $L d l r^{-/} A p o b^{100 / 100}$ in this publication. This model exhibits a lipoprotein profile similar to that of humans with hypercholesterolemia and develops atherosclerosis on a chow diet $(6,7,9)$. Male mice were housed in a pathogen-free barrier facility where they were fed rodent chow containing $4 \%$ fat. Aortic arches isolated at $5(n=7), 10(n=4), 15(n=6)$, $20(n=7), 30(n=4)$ and $40(n=5)$ weeks of age were snap frozen and used for RNA extraction with TRIzol (Invitrogen, Carlsbad, CA, USA), protein lysates and histology. Mouse protocols were approved by the local ethics review board.

Real-time PCR. The aortic arch was carefully dissected and homogenized with FastPrep (Qbiogene, Montreal, Canada). Total-RNA was isolated with an RNeasy mini kit (Qiagen, Valencia, CA, USA). Total-RNA $(0.25 \mu \mathrm{g})$ was reverse transcribed with random primers and Superscript II according to the manufacturer's protocol (Invitrogen). cDNA of $2 \mu \mathrm{l}$ were amplified by real-time PCR with 1X TaqMan universal PCR Master mix (Applied Biosystems, Foster City, CA, USA) in 96-well plates on an ABI 7700 Sequence Detector.

The following assay on Demand kits (Applied Biosystems) were used: MMP-2, Mm00439508; MMP-3, Mm00440295; MMP-7, Mm00487724; MMP-8, Mm00772335, MMP-9, Mm00600163;MMP-11,Mm00485048;MMP-12,Mm00500554; MMP-13, Mm00439491; MMP-14, Mm00485054; lipocalin, Mm00809552; TIMP-1, Mm00441818; TIMP-2, Mm00441825; TIMP-3, Mm00441826; cathepsin K, Mm00484036; cathepsin S, Mm00457902; CD66a, Mm00442360; CD68, Mm00839636; CD11b, Mm00434455; $\alpha$-actin, Mm01187533; SM22 $\alpha$, Mm00441660 and CD3e, Mm00599683. The following genes, ARBP, Mm00725448; cyclophilin D, Mm00835365 and $\beta 2$ microglobulin, Mm00437762 served as RNA loading controls of which ARBP was used, although they all showed similar results.

Histochemical analysis and Oil red $O$ staining. We used picrosirius red staining to examine collagen fibers in the vessels. Acetone-fixed frozen sections of the aortic arch were stained for $1 \mathrm{~h}$ in saturated picric acid containing $0.1 \%$ picrosirius red (Direct Red 80) (Fluka, Buchs, Switzerland). All sections were analyzed under linear polarized light with a Leica DMRB microscope and images were captured with a Leica DC480 color video camera. Thick, mature collagen fibers were orange-red, and thin, immature fibers were green $(10,11)$.

Oil red $\mathrm{O}$ staining was used to detect lipid accumulation in aortic tissue. Formaldehyde-fixed frozen sections of the aortic arch were stained for $15 \mathrm{~min}$ in $0.3 \%$ Oil red $\mathrm{O}$ dissolved in isopropanol and then counterstained with hematoxylin (Vector Laboratories, Burlingame, CA, USA).
Immunohistochemistry. Tissues from the aortic arch were cryosectioned and fixed in acetone. Endogenous peroxidase activity was quenched by treatment with $3 \%$ hydrogen peroxide for $5 \mathrm{~min}$ followed by incubation in 5\% blocking BSA solution. Sections were then incubated with a primary mouse monoclonal anti-mouse $\alpha$-smooth muscle actin antibody (Sigma, St. Louis, MO, USA) or with a primary monoclonal rat anti-mouse CD68 (AbD Serotec, Düsseldorf, Germany) at $4^{\circ} \mathrm{C}$ overnight. Sections were then incubated with a secondary biotinylated goat anti-rat or anti-mouse IgG (Dako Cytomation, Glostrup, Denmark). Avidin-biotin peroxidase complexes were added, followed by visualization with 3,3'-diaminobenzidine tetrahydrochloride (Vector Laboratories). All sections were counterstained with hematoxylin.

In situ zymography. Quenched fluorescein-labeled gelatin (1 mg/ml, DQ gelatin from pig skin) (Molecular Probes, Eugene, OR, USA) was mixed (1:1) with agarose melted in buffer (50 mM Tris- $\mathrm{mCl}, \mathrm{pH} 7.4,10 \mathrm{mM} \mathrm{CaCl}_{2}$, and $0.05 \%$ Brij 35) and incubated on unfixed frozen sections of the aortic arch at $37^{\circ} \mathrm{C}$ for $24 \mathrm{~h}$. The fluorescent area produced by proteolytic digestion of quenched fluorescein-labeled gelatin was recognized as the combined gelatinase activity (MMP-2 and MMP-9). Generation of fluorescent activity was prevented by addition of the global MMP inhibitors 1,10 phenanthroline $(0.4 \mathrm{mM})$ and EDTA $(10 \mathrm{mM})$, which also served as a negative control.

Gel-based zymography. Aortic lysates $(3 \mu \mathrm{g})$ were mixed with native loading buffer 1:1 and separated using a 10\% zymography gel (Invitrogen) to document cleavage of either gelatin by MMP-2 and MMP-9 or of casein by MMP-7, -11, -13 and -13. After electrophoresis the proteins in the gel were renatured and developed (Invitrogen). The gel was thereafter stained with Coomassie Blue and destained until clear. The gelatinolytic activity was evident as clear bands against a dark blue background.

Statistical analysis. Statistical significance was determined with the Mann-Whitney test and $\mathrm{P}<0.05$ was considered statistically significant after using the Bonferroni correction for multiple comparisons.

\section{Results}

Atherosclerotic lesion development is initiated in 15-week-old $\mathrm{Ldlr}^{-1-}$ Apob ${ }^{100 / 100}$ mice. The development of atherosclerosis has previously been evaluated in 10 - to 60-week-old $\mathrm{Ldlr}^{-1} \mathrm{Apob} \mathrm{b}^{100 / 100}$ mice. Lipids started to accumulate in the aortas of 20 -week-old mice (7). We obtained a similar result when investigating lipid accumulation in the aortic arch (Fig. 1). We also included 5-week-old mice in order to analyze protease expression prior to lesion formation. Small amounts of lipids were visible in aortas without lesions in 15-week-old mice, whereas stronger lipid accumulation was first observed in lesions of aortas from 20 -week-old mice. Lipid staining was more pronounced in lesions of 40-week-old mice, as indicated by multiple lesions and simultaneous foam cell formation revealed by Oil red $\mathrm{O}$ staining (Fig. 1). Immunohistochemistry showed pronounced CD68 staining in lesion areas of 20- and 40-week-old mice 

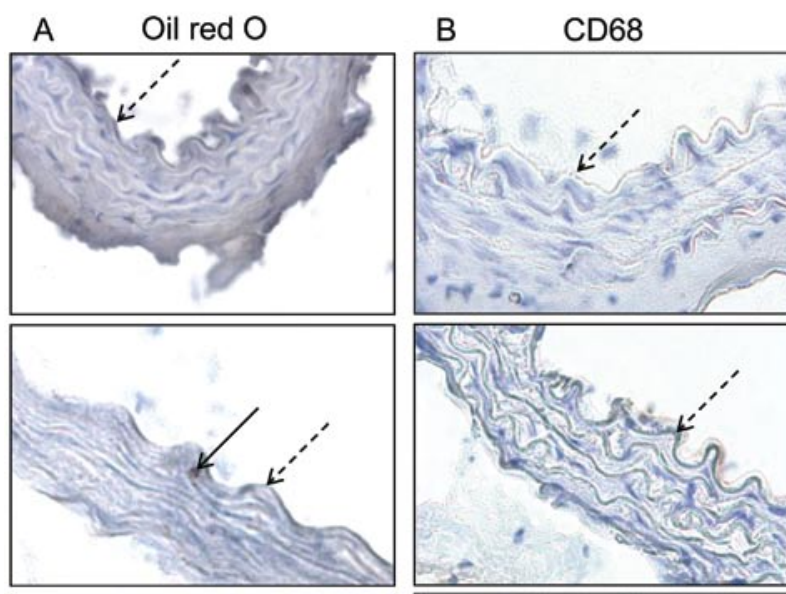

$5 w$
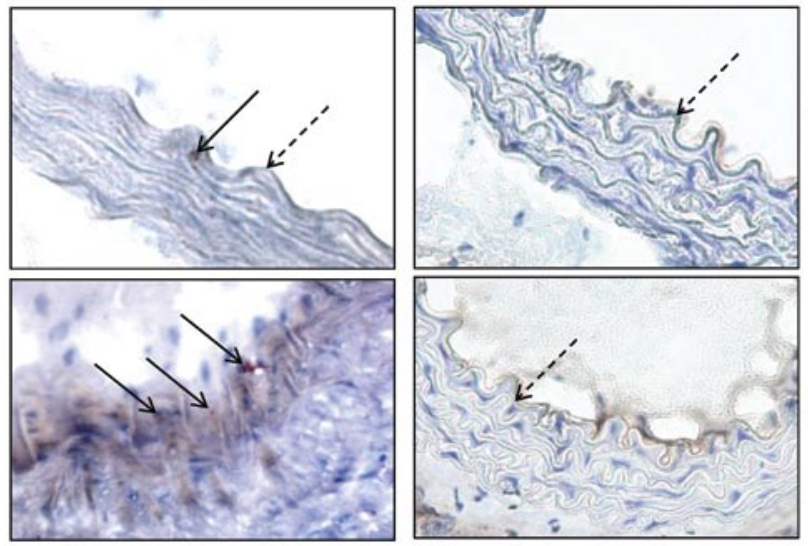

$15 w$
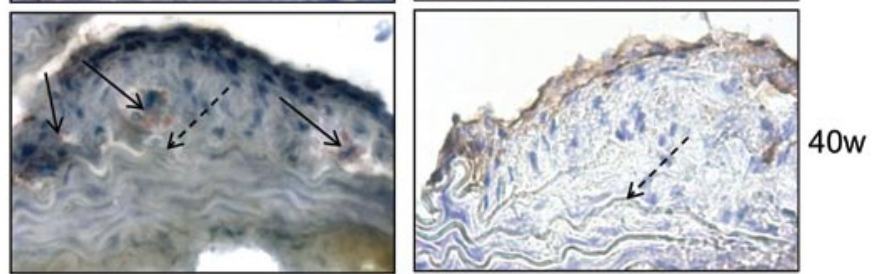

Figure 1. Atherosclerotic lesion development in 5- 15-, 20- and 40-week-old $L d l r^{-1} A p o b^{100 / 100}$ mice. (A) Lipid accumulation was visualized in representative aortas from 5- 15-, 20- and 40-week-old $\mathrm{Ldll}^{-/} \mathrm{Apob}^{100 / 100}$ mice by Oil red O staining. Black arrows indicate positive Oil red $\mathrm{O}$ staining and dotted arrows indicate internal elastic lamina. (B) CD68 staining to visualize macrophages in $L d l r^{-1} A p o b^{100 / 100}$ mice. Magnification, $\mathrm{x} 400$.
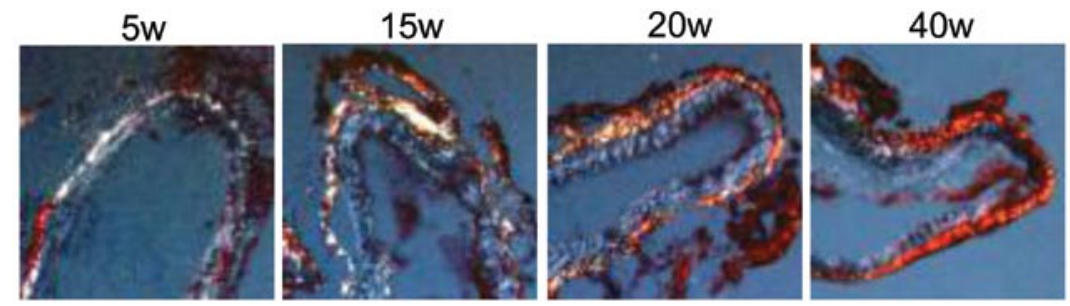

Figure 2. Low levels of immature collagen at later stages of atherosclerotic development. Collagen content was visualized with picrosirius red staining in representative aortas from 5-, 15-, 20- and 40-week-old $\mathrm{Ldlr}^{-1} \mathrm{Apob}^{100 / 100}$ mice. Thick, mature fibers (orange-red) or thin, immature fibers (white-green) are visible under linear polarized light. Magnification, $\mathrm{x} 400$.

but only weak staining at 15 weeks (Fig. 1). There was no CD68 staining prior to 15 weeks.

Furthermore, we performed staining of collagen with picrosirius red to analyze the presence of thick, mature fibers (orange-red) or thin, immature fibers (grey-green) under linear polarized light. We demonstrated that the lesions of 40-week-old mice contained immature collagen in the intima/media layer and mature collagen fibers in the adventitia. The lesion was surrounded by a small stripe of mature collagen (Fig. 2).

Limited protease expression during atherosclerotic development in $\mathrm{Ldll}^{-1-} \mathrm{Apob}^{100 / 100}$ mice. We analyzed the mRNA expression of MMP-2, -3, -7, -8, -9, -11, -12, -13, -14, TIMP-1, -2, -3, cathepsin $\mathrm{K}$, cathepsin $\mathrm{S}$ and lipocalin in the aortic arch by quantitative real-time RT-PCR. No significant changes in the expression of MMP-3, -7, -11, -12, -14, TIMP-2, TIMP-3 or cathepsin $\mathrm{K}$ were observed at the investigated time points (data not shown). MMP-2, MMP-9 and lipocalin were significantly up-regulated in aortas from 30- and 40-week-old mice compared to aortas from 5-week-old mice (Fig. 3A). MMP-2 was barely detectable in aortas of 5-week-old mice. A significant correlation was noted (Fig. 3A, $\mathrm{R}^{2}=0.69$ ) between the expression of MMP-9 and lipocalin. Human lipocalin binds MMP-9 and thereby protects it from degradation (12). In contrast, murine MMP-9 does not contain the cysteine at amino acid 87 necessary for lipocalin binding. The expression of TIMP-1 and cathepsin $\mathrm{S}$ were slightly down-regulated in aortas of 30-week-old mice (Fig. 3B). However, their expression was restored at 40 weeks. Compared to 5-week-old mice, MMP-8 expression was down-regulated in aortas of 30- and 40-week-old 
A
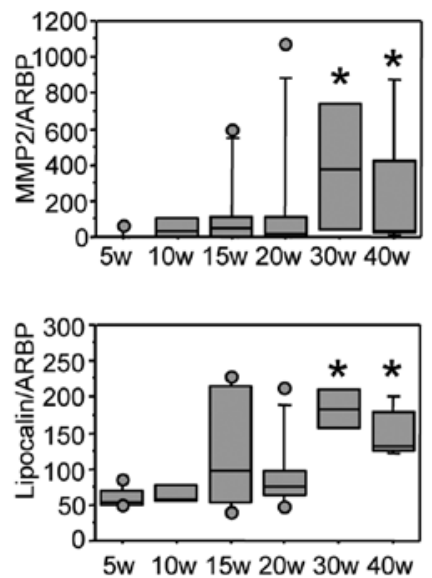

B
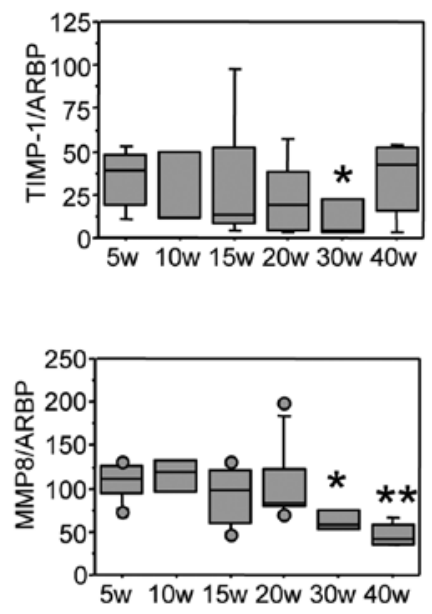

C

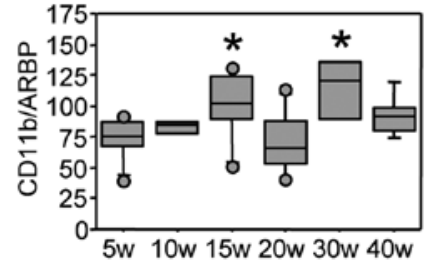

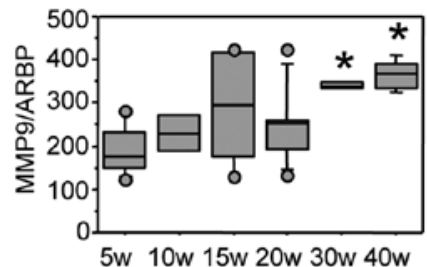
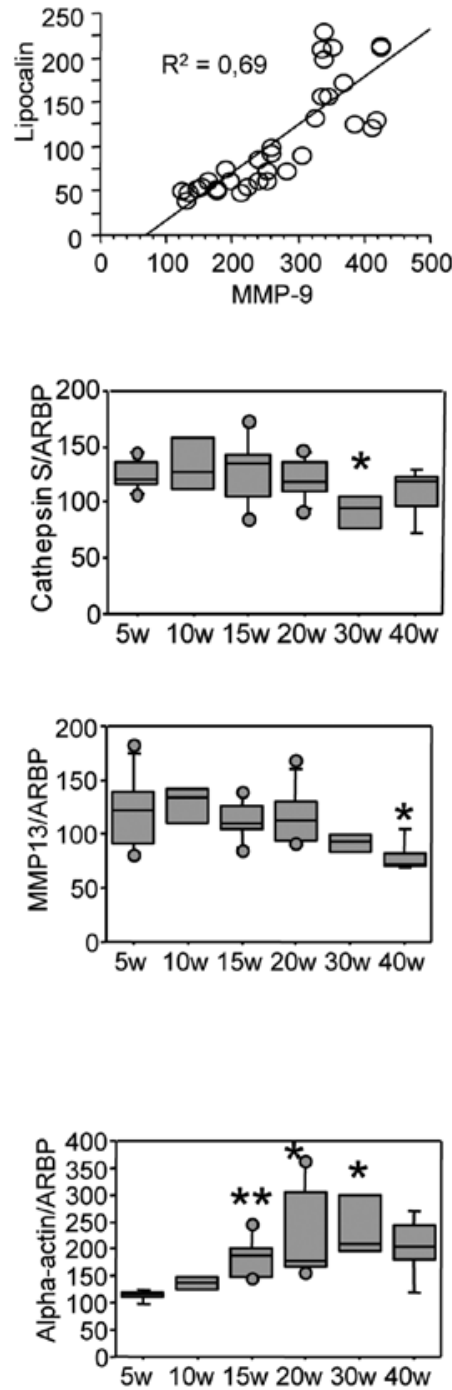

Figure 3. Increased mRNA expression of MMP-2 and MMP-9 at late stages of atherosclerotic development in $L d l r^{-/} A_{p o b} b^{100 / 100}$ mice. Gene expression of MMP genes and MMP-related genes was analyzed by real-time RT-PCR in aortas from 5- to 40-week-old $L_{d l l r^{-1}}$ Apob $^{100 / 100}$ mice. ${ }^{*} \mathrm{P}<0.05$; ${ }^{* *} \mathrm{P}<0.01$. Fiveweek-old (5w) mice $(n=7), 10 w(n=4), 15 w(n=6), 20 w(n=7), 30 w(n=4)$ and 40w $(n=5)$.

mice while MMP-13 expression significantly decreased at 40 weeks (Fig. 3B). The macrophage marker, CD11b, was up-regulated at 15 and 30 weeks with no significant changes at 20 and 40 weeks (Fig. 3C). The smooth muscle cell marker, $\alpha$-actin, increased gradually between 10 and 30 weeks (Fig. 3C). CD66a (neutrophils) and CD3e (T-cells) mRNA could not be detected at any time point (data not shown). Neither $\alpha$-actin nor CD11b expression correlated with any of the MMPs.

Increased gelatinase activity in the aortas of 30-and 40-week-old mice. To investigate whether the increased mRNA expression of MMP-2, MMP-9 and lipocalin resulted in a similar increase in the protein expression and protease activity, we performed gel-based gelatin zymography on aorta lysates (Fig. 4A). In accordance with the mRNA expression, pro-MMP-9 started to increase at 15 weeks, with maximum expression between 30 and 40 weeks. MMP-9 activity followed mRNA and expression of the pro form, i.e., the highest activity occurred after 30 weeks. We could not detect pro-MMP-2 between 5-15 weeks (Fig. 4A). The highest expression of active MMP-2 was observed at 30 and 40 weeks (Fig. 4A and B). Larger complexes of gelatinase activity were mainly detected at 30 and 40 weeks. The compositions of these complexes are not fully clear, although the complex with a molecular weight of $115 \mathrm{kDa}$ probably contains one MMP-9 molecule bound to TIMP-1 (13). In addition, we performed casein zymography to detect the activity of MMP-7, $-11,-12$ and -13 . Only weak or no activity was detected (data not shown). Taken together, the 


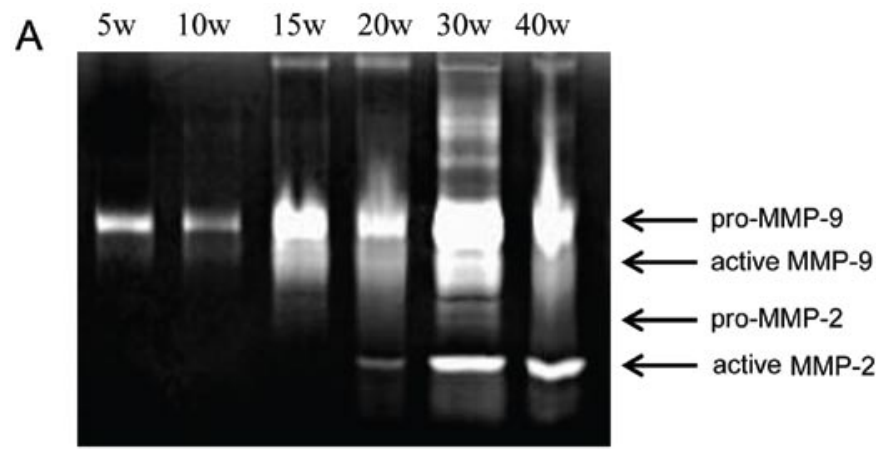

B
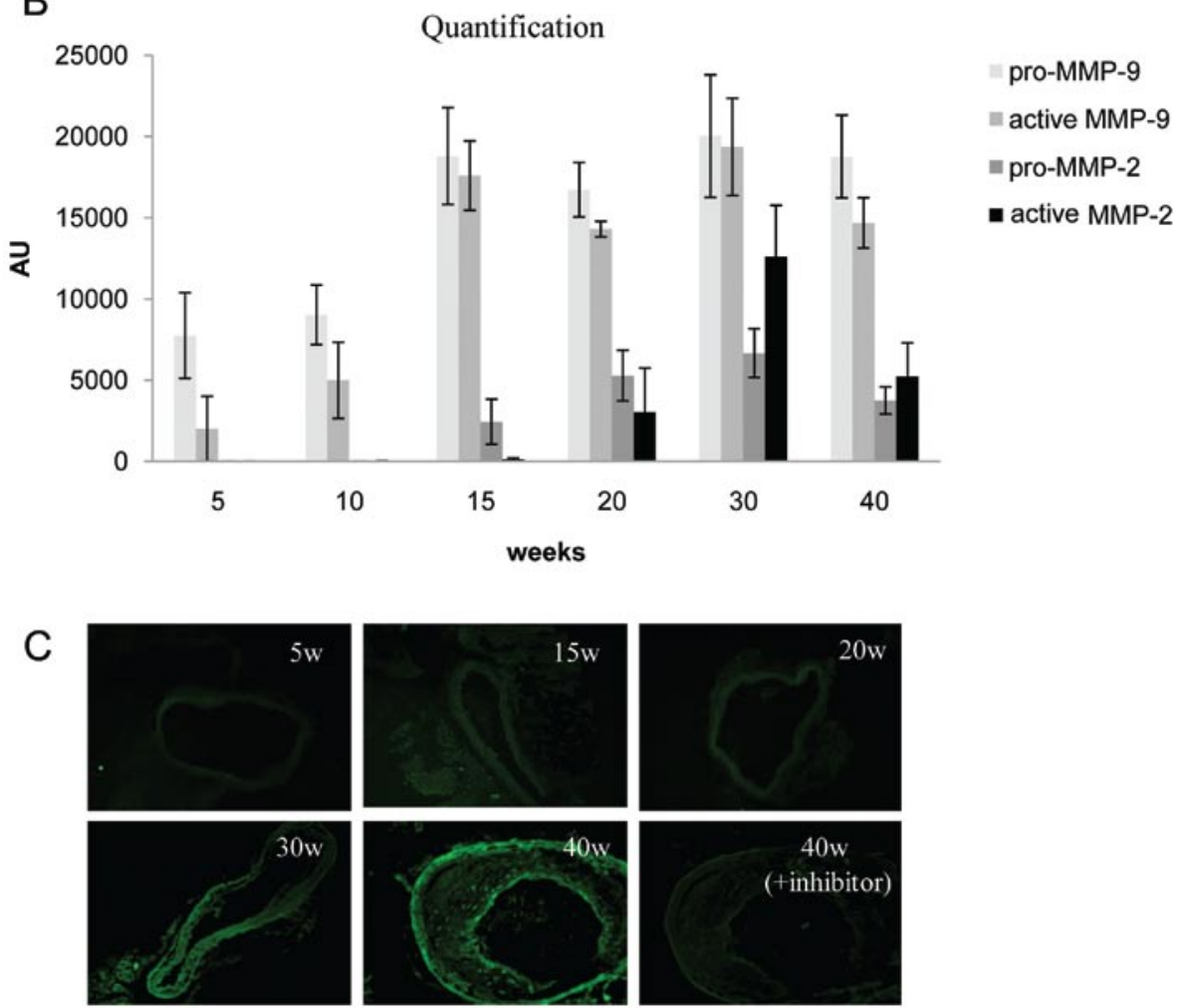

Figure 4. Increased gelatinolytic activity in 30- and 40-week-old $L d l r^{-1} A p o b^{100 / 100}$ mice. (A) Representative gelatin zymography of aorta lysates shows basal expression of the pro form of MMP-9 in 5- and 10-week-old $L d l r^{-1} A_{p o b} b^{100 / 100}$ mice, which increases at 15 weeks with a simultaneous activation of MMP-9 up to 40 weeks. Pro and active forms of MMP-2 are detected in aorta lysates from 20 -week-old mice with the highest expression at 30 and 40 weeks. (B) Quantification of four different gels. (C) Gelatin in situ zymography shows low expression of the gelatinases MMP-2 and MMP-9 in frozen sections of aortas from 5- 15- and 20-week-old $\mathrm{Ldll}^{-1} \mathrm{Apob} \mathrm{b}^{100 / 100}$ mice with the highest expression in 30-week-old and 40-week-old mice. The global MMP inhibitors, 1,10 phenanthroline $(0.4 \mathrm{mM})$ and EDTA $(10 \mathrm{mM})$, served as negative controls. Magnification, $\mathrm{x} 400$.

protein data show an expression pattern similar to that of the mRNA data.

We performed gelatin in situ zymography to verify and localize active MMP-2 and MMP-9, i.e., to identify gelatinase activity in the aorta. Gelatinase activity was weak in aortas from young mice, whereas tissue from 30- and 40-week-old mice had substantial gelatinolytic activity (Fig. 4C). Gelatinolytic activity was observed in the adventitia and intima layers, the latter probably corresponding to the subendothelial macrophages found as indicated in Fig. 1B.

\section{Discussion}

In the present study, we investigated the expression and activity of several matrix-degrading proteases before and during early stages of lesion formation in $\mathrm{Ldlr}^{-1-} \mathrm{Apob}^{100 / 100}$ mice. The pure $L d l r^{-/}$mice only develop tiny atherosclerotic lesions on a normal chow diet and thus require a high-fat diet in order to develop severe atherosclerotic lesions. ApoE ${ }^{-/-}$mice have an increased plasma VLDL and very early lesion development, which is not observed in humans. ApoE ${ }^{-/-}$mice develop atherosclerotic lesions as early as at 15-20 weeks. The situation with $L d l r^{-1-} A p o b^{100 / 100}$ mice resembles a plasma lipid profile that is similar to humans with familiar hypercholesterolemia. In addition, the $\mathrm{Ldlr}^{-/} \mathrm{Apob} \mathrm{b}^{100 / 100}$ mouse model develops atherosclerosis on a chow diet at later stages, i.e. at 30-40 weeks. In the present study, we demonstrated that gelatinases appear to be the main proteolytic enzymes active during atherogenesis in $\mathrm{Ldlr}^{-1-} \mathrm{Apob} \mathrm{b}^{100 / 100}$ mice. Gel-based gelatin zymography detected MMP-9 activity in aortas of 15 week-old mice, at which time point lipid accumulation was observed, but without lesions or macrophages. MMP-2 was first expressed at 20 weeks. MMP-2 
and MMP-9 expression increased by age and were highest in aortas from 30- and 40-week-old mice, which corresponded with macrophage staining and pronounced lesion development. In contrast, the expression of MMP-8, MMP-13 and TIMP-1 were slightly down-regulated during the later stages of atherogenesis. All other investigated proteases showed very low expression or did not change over time. Taken together, the data show that there is very low proteolytic expression at early stages of atherogenesis and that there is a limited repertoire of proteolytic enzymes associated with the progression of atherosclerosis in this mouse model.

It has been previously shown that there are common signaling pathways regulating MMP expression. We have shown that during differentiation of monocytes to macrophages, the expression of a range of proteinases and their inhibitors was up-regulated in the human THP-1 cell line (14). Of the 56 proteinases/inhibitors investigated, 17 were up-regulated during macrophage differentiation. MMP-2 and -9 , in addition to several other MMPs and cathepsins, were among the up-regulated proteases. Most MMPs share a limited number of promoter elements, and a few transcription factors could be responsible for the concomitant expression of proteases induced by monocyte differentiation (15). However, in the present study, only MMP-2 and -9 were up-regulated and no coordinated regulation of protease expression was detected, which could be due to the relatively low increase of macrophages seen in this model. Of note, intimal and adventitial areas appeared to be the main cellular sources of MMP-2 and MMP-9 activity in the $L d l r^{--} A p o b^{100 / 100}$ mice based on in situ zymography analyses. Intimal macrophages most likely correspond to the intimal activity of MMP-2 and MMP9 observed herein. Interestingly, we recently reported that expression of subtype 4 of a disintegrin and metalloproteinase with thrombospondin motifs (ADAMTS4) was increased during disease progression in $\mathrm{Ldlr}^{-/} \mathrm{Apob}^{100 / 100}$ mice and in human atherosclerotic lesions which were co-localized to macrophage-rich areas and could argue for the importance of other metalloproteinases in this model (8).

Gelatin in situ zymography showed increased expression of MMP-2 and MMP-9 in aortas from 30- and 40-week-old mice. Lipocalin, which may protect MMP-9 from cleavage in humans and promote its activation, also showed its highest expression in aortic lysates from 30- and 40-week-old mice. However, murine MMP-9 does not bind lipocalin. Nevertheless, we observed a strong association between the mRNA expression of MMP-9 and lipocalin, indicating that they may be similarly regulated during atherogenesis. At 30-40 weeks there was also a decrease in the TIMP-1 expression, which together with the effects of lipocalin may reflect the high MMP-9 activation at the observed time points.

Surprisingly, mRNA expression of cathepsin S was not increased as we expected, which is in contrast to our previous results in $A p o E$-deficient mice that showed a dramatic increase in its expression in 10 - and 20 -week-old animals (16). The mRNA expression was strongly associated to the macrophage marker CD68, which again was only weakly increased in the Ldlr $^{-1-}$ Apob $^{100 / 100}$ mice. Also, Sukhova et al showed that the double knockout of cathepsin $\mathrm{S}^{-/} \mathrm{Ldlr}^{-/}$mice developed significantly less atherosclerosis, as indicated by plaque size and stage of development (17). The discrepancy between our results and data obtained using the other models may be explained by the differences in LDL/VLDL-induced response on cathepsin regulation.

There are several mechanisms whereby the activity of MMPs can affect the vessel wall cells. Some of the more important mechanisms include the regulation of migration, proliferation and apoptosis of smooth muscle cells and the sequestration of signaling molecules, such as growth factors and growth factor-binding proteins, all important key steps in atherogenesis (18). We detected very little protease expression at earlier time points, i.e., stages preceding lesion formation. Minor, local expression could, however, not be ruled out. MMP-12, a macrophage elastase, was constitutively expressed during development. Yamada et al have showed that MMP-12 transgenic rabbits accelerated the initiation of atherosclerosis and stimulated the progression of fatty streaks to fibrous plaques (19). Johnson et al have reported that brachiocephalic artery plaques were significantly larger in ApoE/MMP-3 and ApoE/MMP-9 double knockouts compared to controls. The lesion size was reduced in ApoE/MMP-12 double knockouts compared to controls, and double knockouts had increased smooth muscle cell and reduced macrophage content in the plaque. MMP-9 appears to play a protective role, limiting plaque growth and promoting a stable plaque phenotype, while MMP-12 supports lesion expansion and destabilization (3). Expression of the collagenases MMP-8 and MMP-13 appeared to decrease at the later time points, which could explain the relatively high amount of collagen observed in the vessels and surrounding the lesion in this model.

In conclusion, results using $L d l r^{-/-} A p o b^{100 / 100}$ mice suggest that MMP-2 and MMP-9 are the main proteases involved during atherogenesis in this mouse model. Taken together, the data show that there is very low proteolytic expression at early stages of atherogenesis and that there is a limited repertoire of proteolytic enzymes associated with the progression of atherosclerosis in this mouse model. The lesion development is initiated by infiltrated macrophages that mainly produce MMP-2 and MMP-9.

\section{Acknowledgements}

This study was supported by the Swedish Research Council (12660, 20749), the Swedish Heart-Lung Foundation, the European Commission (FAD, Health-F2-2008-200647), the Nanna Svartz Foundation, Loo and Hans Ostermans Foundation, the Lars Hierta Memorial Foundation, Anders Otto Swärds Foundation/Ulrika Eklunds Foundation and the Gun and Bertil Stohnes Foundation.

\section{References}

1. Hansson GK, Robertson AK and Soderberg-Naucler C: Inflammation and atherosclerosis. Annu Rev Pathol 1: 297-329, 2006.

2. Newby AC: Dual role of matrix metalloproteinases (matrixins) in intimal thickening and atherosclerotic plaque rupture. Physiol Rev 85: 1-31, 2005.

3. Johnson JL, George SJ, Newby AC and Jackson CL: Divergent effects of matrix metalloproteinases $3,7,9$, and 12 on atherosclerotic plaque stability in mouse brachiocephalic arteries. Proc Natl Acad Sci USA 102: 15575-15580, 2005.

4. Nilsson L, Jonasson L, Nijm J, Hamsten A and Eriksson P: Increased plasma concentration of matrix metalloproteinase-7 in patients with coronary artery disease. Clin Chem 52: 1522-1527, 2006. 
5. Blankenberg S, Rupprecht HJ, Poirier O, Bickel C, Smieja M, Hafner G, Meyer J, Cambien F and Tiret L: Plasma concentrations and genetic variation of matrix metalloproteinase 9 and prognosis of patients with cardiovascular disease. Circulation 107: 1579-1585, 2003.

6. Farese RV Jr, Veniant MM, Cham CM, Flynn LM, Pierotti V, Loring JF, Traber M, Ruland S, Stokowski RS, Huszar D and Young SG: Phenotypic analysis of mice expressing exclusively apolipoprotein B48 or apolipoprotein B100. Proc Natl Acad Sci USA 93: 6393-6398, 1996.

7. Skogsberg J, Lundstrom J, Kovacs A, Nilsson R, Noori P, Maleki S, Kohler M, Hamsten A, Tegner J and Bjorkegren J: Transcriptional profiling uncovers a network of cholesterol-responsive atherosclerosis target genes. PLoS Genet 4: e1000036, 2008.

8. Wagsater D, Bjork H, Zhu C, Bjorkegren J, Valen G, Hamsten A and Eriksson P: ADAMTS- 4 and -8 are inflammatory regulated enzymes expressed in macrophage-rich areas of human atherosclerotic plaques. Atherosclerosis 196: 514-522, 2008

9. Lieu HD, Withycombe SK, Walker Q, Rong JX, Walzem RL, Wong JS, Hamilton RL, Fisher EA and Young SG: Eliminating atherogenesis in mice by switching off hepatic lipoprotein secretion. Circulation 107: 1315-1321, 2003.

10. Deguchi JO, Aikawa E, Libby P, Vachon JR, Inada M, Krane SM, Whittaker P and Aikawa M: Matrix metalloproteinase-13/ collagenase-3 deletion promotes collagen accumulation and organization in mouse atherosclerotic plaques. Circulation 112: 2708-2715, 2005.

11. Luque EH, Angulo E and Montes GS: A histochemical and electron microscopic study on the collagen of nerves in the domestic fowl. J Anat 137: 171-176, 1983.

12. Yan L, Borregaard N, Kjeldsen L and Moses MA: The high molecular weight urinary matrix metalloproteinase (MMP) activity is a complex of gelatinase B/MMP-9 and neutrophil gelatinaseassociated lipocalin (NGAL). Modulation of MMP-9 activity by NGAL. J Biol Chem 276: 37258-37265, 2001.
13. Monier F, Surla A, Guillot M and Morel F: Gelatinase isoforms in urine from bladder cancer patients. Clin Chim Acta 299: $11-23,2000$.

14. Whatling C, Bjork H, Gredmark S, Hamsten A and Eriksson P Effect of macrophage differentiation and exposure to mildly oxidized LDL on the proteolytic repertoire of THP-1 monocytes. J Lipid Res 45: 1768-1776, 2004.

15. Yan C and Boyd DD: Regulation of matrix metalloproteinase gene expression. J Cell Physiol 211: 19-26, 2007.

16. Jormsjo S, Wuttge DM, Sirsjo A, Whatling C, Hamsten A, Stemme S and Eriksson P: Differential expression of cysteine and aspartic proteases during progression of atherosclerosis in apolipoprotein E-deficient mice. Am J Pathol 161: 939-945, 2002.

17. Sukhova GK, Zhang Y, Pan JH, Wada Y, Yamamoto T, Naito M, Kodama T, Tsimikas S, Witztum JL, Lu ML, Sakara Y, Chin MT, Libby P and Shi GP: Deficiency of cathepsin S reduces atherosclerosis in LDL receptor-deficient mice. J Clin Invest 111: 897-906, 2003

18. Sternlicht MD and Werb Z: How matrix metalloproteinases regulate cell behavior. Annu Rev Cell Dev Biol 17: 463-516, 2001.

19. Yamada S, Wang KY, Tanimoto A, Fan J, Shimajiri S, Kitajima S, Morimoto M, Tsutsui M, Watanabe T, Yasumoto K and Sasaguri Y: Matrix metalloproteinase 12 accelerates the initiation of atherosclerosis and stimulates the progression of fatty streaks to fibrous plaques in transgenic rabbits. Am J Pathol 172: 1419-1429, 2008. 